\title{
Global Optimization of Multiscenario Mixed Integer Nonlinear Programming Models Arising in the Synthesis of Integrated Water Networks under Uncertainty
}

\author{
Ramkumar Karuppiah and Ignacio E. Grossmann* \\ Department of Chemical Engineering, Carnegie Mellon University, Pittsburgh, \\ PA 15213, USA.
}

The problem of optimal synthesis of an integrated water system is addressed in this work, where water using processes and water treatment operations are combined into a single network such that the total cost of designing the network and operating it optimally is globally minimized. The network design has to be feasible and optimal over a given set of scenarios in which different operational conditions hold. We propose a superstructure whose optimization is formulated as a multiscenario non-convex Mixed Integer NonLinear Programming (MINLP) problem. A Lagrangean decomposition based algorithm is proposed for the global optimization of such large multiscenario models. An example is presented for the global optimization of an integrated network operating under uncertainty using the proposed algorithm.

Keywords: Global optimization; Integrated water networks; Uncertainty

\section{INTRODUCTION}

Process synthesis under uncertainty is in general a very challenging problem. A number of parameters usually change during the operation of a process network and for which the data is not known exactly. The major objective when synthesizing a network operating under uncertainty is that the design should be optimal and feasible over a range of values of the uncertain parameters. The problem of ensuring feasibility of design has been addressed by Grossmann et al. [1] where the control variables in the system can be adjusted for the parameter changes. In a stochastic programming based approach, the emphasis is on achieving optimality accounting for the fact that the recourse variables can be adjusted for each parameter realization (see Acevedo and Pistikopolous [2] and Liu and Sahinidis [3]). A recent review of the major techniques for optimization under uncertainty is given in Sahinidis [4].

This paper addresses the optimization of integrated water networks operating under uncertain operational conditions. We pose the design problem as a two stage stochastic program formulating it as a deterministic multiscenario Mixed Integer Non-Linear Programming (MINLP) problem since the uncertain parameters can take on a finite number of realizations. An algorithm is proposed to solve the problem to global optimality. We present an example to illustrate 
that the algorithm solves the problem in significantly less time than the MINLP solver BARON (Sahinidis [5]).

\section{PROBLEM STATEMENT}

In this work, we consider the optimal synthesis of an integrated water network consisting of water using process units, water treating units and mixers and splitters, operating under uncertain operational conditions. The amounts of contaminants generated in the process units and the contaminant removal ratios in the treatment units are the uncertain parameters which take different values in each scenario. The objective is to synthesize a network such that the costs of designing the network and the expected cost of operating the network optimally over all scenarios is minimized.

The first stage costs include the investment cost for piping which depends on the maximum flowrate allowable in a pipe, and the design cost of each treatment unit, which is dependent on the maximum flow of wastewater handled by that treatment unit. The operating costs of the network appear in the second stage, which include the cost of obtaining freshwater for use in the process units, the cost of pumping a certain flow of water through the pipes (which should be less than the maximum flow allowable in the pipes) and the operating costs of treating wastewater in the treatment units. The synthesis problem is formulated as a multiscenario non-convex MINLP which is solved to global optimality.

\section{MODEL}

We extend the non-convex NLP formulation for the synthesis of integrated water networks given in Karuppiah and Grossmann [6] to construct the multiscenario MINLP model. A detailed nomenclature of the terms used here is given in [6].

\section{Objective function:}

$\min \phi=A R\left[\sum_{i}\left(C_{p}^{i} y^{i}+I P^{i}\left(\hat{F}^{i}\right)^{\delta}\right)\right]+H \sum_{n} p_{n} \sum_{i} P M^{i} F_{n}^{i}+H \sum_{n} p_{n} C_{F W} F W_{n}+A R \sum_{\substack{t \in T U \\ i \epsilon_{\text {out }}}} I C^{t}\left(\hat{F}^{i}\right)^{\alpha}+H \sum_{n} p_{n} \sum_{\substack{t \in T U \\ i \epsilon_{\text {out }}}} O C F_{n}^{i}$

Here, $p_{n}$ is the probability assigned to scenario $n, C_{p}^{i}$ is the cost coefficient corresponding to existence of pipe $i, I P^{i}\left(\hat{F}^{i}\right)^{\delta}$ is the investment cost of a pipe $i$, while $P M^{i} F_{n}^{i}$ is the cost of pumping water inside a pipe $i$ in scenario $n$. The design variable $y^{i}$ pertains to the existence of a stream/pipe $i$. The vector $\hat{F}^{i}$ is the set of first stage design variables which pertains to the maximum

flows allowable in the pipes while the vector $F_{n}^{i}$ is the set of second stage state variables which correspond to the flows in the pipes in each scenario $n$.

\section{Mixer Units:}

$$
\begin{aligned}
& F_{n}^{k}=\sum_{i \in m_{\text {in }}} F_{n}^{i} \quad \forall m \in M U, k \in m_{\text {out }}, \forall n \in N \\
& F_{n}{ }^{k} C_{j n}^{k}=\sum_{i \in m_{\text {in }}} F_{n}^{i} C^{i}{ }_{j n}^{i} \quad \forall j, \forall m \in M U, k \in m_{\text {out }}, \forall n \in N
\end{aligned}
$$


Here $C_{j n}^{i}$ is the concentration of contaminant $j(\mathrm{ppm})$ in stream $i$ in scenario $n$.

\section{Splitter Units:}

$$
\begin{array}{ll}
F_{n}{ }^{k}=\sum_{i \in s_{\text {out }}} F_{n}{ }_{n}^{i} & \forall s \in S U, k \in s_{\text {in }}, \forall n \in N \\
C_{j n}^{i}=C_{j n}^{k} & \forall j, \forall s \in S U, \forall i \in s_{\text {out }}, k \in s_{\text {in }}, \forall n \in N
\end{array}
$$

Process Units:

$$
\begin{aligned}
& F_{n}^{k}=F_{n}^{i}=P^{p} \quad \forall p \in P U, i \in p_{\text {in }}, k \in p_{\text {out }}, \forall n \in N \\
& P^{p} C_{j n}^{i}+L_{j n}^{p} \times 10^{3}=P^{p} C_{j n}^{k} \quad \forall j, \forall p \in P U, i \in p_{\text {in }}, k \in p_{\text {out }}, \forall n \in N
\end{aligned}
$$

The contaminant load of contaminant $j$ inside a process unit $p$ for each scenario $n$ is different and is given by $L_{j n}^{p}$.

\section{Treatment Units:}

$F_{n}{ }^{k}=F_{n}^{i} \quad \forall t \in T U, i \in t_{\text {out }}, k \in t_{\text {in }}, \forall n \in N$

$C_{j n}^{i}=\beta_{j n}^{t} C_{j n}^{k} \quad \forall j, \forall t \in T U, i \in t_{\text {out }}, k \in t_{\text {in }}, \forall n \in N$

The contaminant removal ratios in the treatment units are different in each scenario $n$ and so $\beta_{j}^{t}$ which is defined as $\beta_{j}^{t}=1-\{$ (Removal ratio for contaminant $j$ in unit $t$ (in \%)) / 100\} takes on different values $\left(\beta_{j n}^{t}\right)$ in each scenario $n$.

\section{Bound strengthening cuts:}

$\sum_{p \in P U} L_{j n}^{p} \times 10^{3}=\sum_{\substack{t \in T U \\ k \in t_{\text {in }}}}\left(1-\beta_{j n}^{t}\right) F_{n}^{k} C_{j n}^{k}+F_{n}^{\text {out }} C_{j n}^{\text {out }} \quad \forall j, \forall n \in N$

\section{Design Constraints:}

$\hat{F}^{i L} y^{i} \leq \hat{F}^{i} \leq \hat{F}^{i U} y^{i} \quad \forall i$

Linking constraints: The "hard" constraints that link the variables of each scenario with the design variables are given in eq (12).

$\hat{F}^{i} \geq F_{n}{ }^{i} \quad \forall i, \forall n \in N$

The multiscenario MINLP model (P) comprises equations (1) - (12).

\section{SOLUTION STRATEGY}

The multiscenario models grow quickly in size with the number of scenarios and are very difficult to solve to global optimality without the help of specialized techniques. A decomposition scheme is proposed for the generation of tight lower bounds (within a branch and bound setting), where we use Lagrangean relaxation to decompose the model (P) into single scenario subproblems that are solved to global optimality. A heuristic is used for the generation of good upper bounds. These lower and upper bounds are converged within a specified tolerance in a spatial branch and bound algorithm.

4.1. Generation of tight lower bounds: In order to construct a Lagrangean relaxation of the original MINLP problem, we dualize the linking constraints (eq (12)) between the different scenarios. To do this, we create copies of the design variables $\hat{F}^{i}$ and $y^{i}$ for each scenario, given by $\hat{F}_{n}^{i}$ and $y_{n}^{i}$ respectively, 
and replace $\hat{F}^{i}$ and $y^{i}$ by these newly created variables in model (P). Hence, eqs (11) and (12) get modified to yield eqs (13) and (14) respectively.

$$
\begin{aligned}
& \hat{F}^{i L} y_{n}^{i} \leq \hat{F}_{n}^{i} \leq \hat{F}^{i U} y_{n}^{i} \quad \forall i, \forall n \in N \\
& \hat{F}_{n}{ }^{i} \geq F_{n}{ }^{i} \quad \forall i, \forall n \in N
\end{aligned}
$$

The objective function is also altered as shown in eq (15), where $\hat{F}^{i}$ in the original objective function is replaced by $\hat{F}_{1}^{i}$.

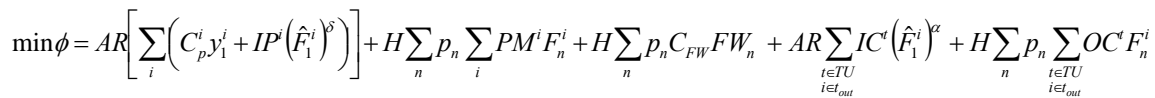

Finally, we add eqs (16) and (17) to (P) to get a reformulated model (RP).

$\hat{F}_{n}^{i}-\hat{F}_{n+1}^{i}=0 \quad \forall i, \forall n \in N, n<|N|$

$y_{n}^{i}-y_{n+1}^{i}=0 \quad \forall i, \forall n \in N, n<|N|$

Further, we multiply the eqs (16) and (17) with $\lambda_{i n}^{f}(\forall n \in N, n<|N|)$ and $\lambda_{\text {in }}^{y}(\forall n \in N, n<|N|)$ respectively and transfer these constraints to the objective function to get a Lagrangean relaxation of the original problem (P), which is denoted by (LRP) and is decomposable into smaller sub-problems that are easier to solve. The parameters $\lambda_{i n}^{f}$ and $\lambda_{i n}^{y}$ are known as Lagrange multipliers. The model (LRP) is then decomposed into $|N|$ smaller models that contain variables pertaining to only one scenario. It is to be noted that the bounds of the variables in all the sub-problems are the same as in the original problem. A set of decomposed problems is as follows:

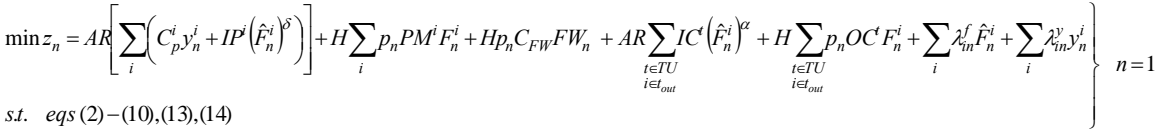

$$
\begin{aligned}
& \left.\begin{array}{l}
\min z_{n}=H \sum_{i} p_{n} P M^{i} F_{n}^{i}+H p_{n} C_{F W} F W_{n}+H \sum_{\substack{i \in T \\
i \in t_{\text {ton }}}} p_{n} O C^{t} F_{n}^{i}+\sum_{i}\left(\lambda_{i n}^{f}-\lambda_{i(n-1)}^{f}\right) \hat{F}_{n}^{i}+\sum_{i}\left(\lambda_{i n}^{y}-\lambda_{i(n-1)}^{y}\right) y_{n}^{i} \\
\text { s.t. eqs }(2)-(10),(13),(14)
\end{array}\right\} n=2 \ldots|N|-1
\end{aligned}
$$

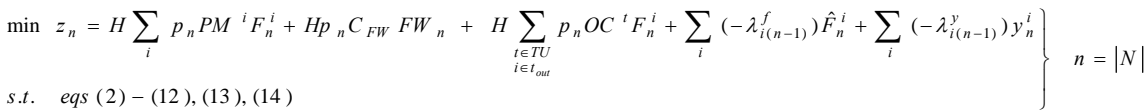

Each of these sub-problems is globally minimized to obtain a solution $z_{n}^{*}$. The sum $\sum_{n \in N} z_{n}^{*}$ yields a valid lower bound to the solution of (P). Instead of using such a lower bound we generate valid cuts in the space of the original design and state variables based on the solutions $z_{n}^{*}$, which are given in eqs (18) - (20).

$$
z_{n}^{*} \leq A R\left[\sum_{i}\left(C_{p} y^{i}+I P^{i}\left(\hat{F}^{i}\right)^{\delta}\right)\right]+H \sum_{i} p_{n} P M F_{n}^{i}+H p_{n} C_{F W} F W_{n}+A R \sum_{\substack{t \in T U \\ i \epsilon_{\text {out }}}} I C\left(\hat{F}^{i}\right)^{\alpha}+H \sum_{\substack{t \in \in U \\ i \theta_{\text {out }}}} p_{n} O C F_{n}^{i}+\sum_{i} \lambda_{i n}^{f} \hat{F}^{i}+\sum_{i} x_{i n}^{u} y^{i} \quad n=1
$$




$$
\begin{aligned}
& \left.z_{n}^{*} \leq H \sum_{i} p_{n} P M^{i} F_{n}^{i}+H p_{n} C_{F W} F W_{n}+H \sum_{\substack{t \in T U \\
i \epsilon_{\text {out }}}} p_{n} O C F_{n}^{i}+\sum_{i}\left(\lambda_{i n}^{f}-\lambda_{i(n-1)}^{f}\right)\right)^{i}+\sum_{i}\left(\lambda_{i n}^{y}-\lambda_{i(n-1)}^{v}\right) y^{i} \quad n=2 \ldots .|N|-1 \\
& z_{n}^{*} \leq H \sum_{i} p_{n} P M^{i} F_{n}^{i}+H p_{n} C_{F W} F W_{n}+H \sum_{\substack{t \in \in U \\
i \in t_{\text {out }}}} p_{n} O C^{i} F_{n}^{i}+\sum_{i}\left(-\lambda_{i(n-1)}^{f}\right) \hat{F}^{i}+\sum_{i}\left(-\lambda_{i(n-1)}^{v}\right) y^{i} \quad \begin{array}{r}
(19) \\
n=|N|
\end{array}
\end{aligned}
$$

These cuts are then added to the model (P). Futhermore, the Lagrange multipliers can be updated using sub-gradient methods so as to derive additional cuts, in the same way as before, to add to the original problem (P). The initial values of the Lagrange multipliers are chosen arbitrarily. This procedure of updating the multipliers and adding cuts is arbitrary and can be performed any number of times. The problem $(\mathrm{P})$ with these cuts added is convexified by constructing convex envelopes for the non-convex nonlinear terms and the resulting MILP (model (R)) is solved to predict a valid lower bound to the solution of $(\mathrm{P})$.

4.2. Upper bound generation: A heuristic procedure is used to generate upper bounds at every node of the branch and bound tree. We solve the single scenario model (obtained from (P) by taking a single element in the set $\mathrm{N}$ ) for all the given scenarios $n \in \mathrm{N}$ to global optimality, and superimpose the resulting structures. We fix the design variable $y^{i}$ to 1 if there exists a non-zero flow $F_{n}^{i}$ in the solution of at least one of the $|N|$ single scenario sub-problems. The problem (P) is transformed from a non-convex MINLP to a non-convex NLP which is solved to get an upper bound.

\section{NUMERICAL EXAMPLE}

We consider a network consisting of two water processing units and two water treatment units whose superstructure is shown in Fig. 1.

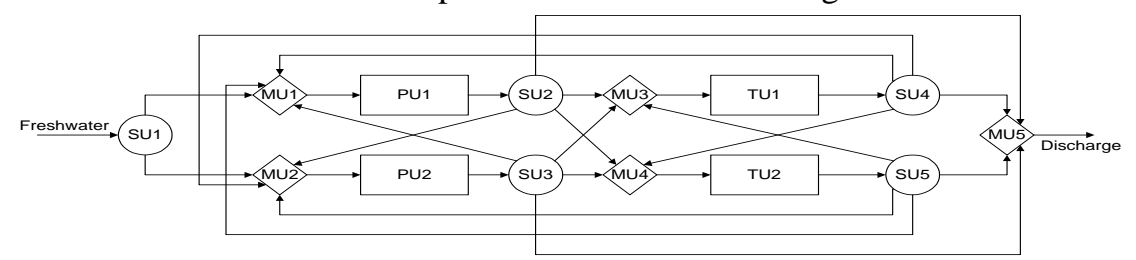

Fig. 1 Superstructure of a 2 Process unit -2 Treatment unit integrated network

It is a system involving two contaminants $\mathrm{A}$ and $\mathrm{B}$, which are generated in the process units and removed using the treatment units. The concentration of these pollutants has to be reduced to less than $10 \mathrm{ppm}$ in the effluent stream discharged into the environment. This system operates over a set of 10 scenarios in one year, where the uncertainties correspond to the contaminant loads in the process units and the contaminant removal ratios in the treatment units. The data used for optimizing this integrated water network can be obtained from the authors. This multiscenario MINLP corresponding to this example involves 28 binary variables, 868 continuous variables, 1044 constraints and 490 non- 
convex terms and was initially solved using GAMS/BARON 7.2 on an Intel 3.2 $\mathrm{GHz}$ machine with $1 \mathrm{~GB}$ memory. The termination criterion used was that the gap between the upper and lower bounds should be less than the specified tolerance of $1 \%$. On directly using BARON to solve the problem, it could not verify global optimality of the upper bound it generated in more than 10 hours. The application of the proposed algorithm yields an expected cost of $\$ 651,653.06$, which is the global solution to the problem. It is also found that the lower and upper bounds converge to within the specified tolerance at the root node of the branch and bound tree. The proposed algorithm takes a total of 62.8 CPUsecs to solve which is drastically less than the time taken by BARON to optimize the original model. The optimal network topology is shown in Fig. 2 where, alongside the pipe connections, the maximum flowrates that can be handled by the pipes are shown.

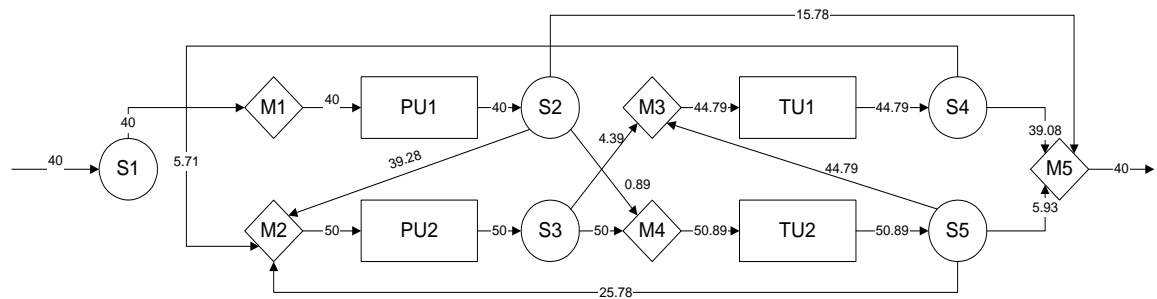

Fig. 2 Optimal solution for a 2 Process unit -2 Treatment unit system operating under uncertainty

Acknowledgement. The authors gratefully acknowledge financial support from the National Science Foundation under Grant CTS-0521769.

\section{REFERENCES}

1. I.E. Grossmann, K.P. Halemane and R.E. Swaney, Computers and Chemical Engineering, 7(1983) 439.

2. J. Acevedo and E.N. Pistikopolous, Computers and Chemical Engineering, 22(1998) 647.

3. M.L. Liu and N.V. Sahinidis, Industrial and Engineering Chemistry Research, 35(1996) 4154.

4. N.V. Sahinidis, Computers and Chemical Engineering, 28(2004) 971.

5. N. Sahinidis, Journal of Global Optimization, 8(1996) 201.

6. R. Karuppiah and I.E. Grossmann, Global Optimization for the Synthesis of Integrated Water Systems in Chemical Processes, submitted to Computers and Chemical Engineering (2005). 